\title{
Solvability of a Class of Operator-Differential Equations of Third Order with Complicated Characteristic on the Whole Real Axis
}

\author{
Abdel Baset I. Ahmed, Mohamed A. Labeeb \\ Egyptian Russian University, Badr City, Egypt \\ Email: abdel2007@yandex.ru, m.labeeb85@yahoo.com
}

How to cite this paper: Ahmed, A.B.I. and Labeeb, M.A. (2018) Solvability of a Class of Operator-Differential Equations of Third Order with Complicated Characteristic on the Whole Real Axis. Open Access Library Journal, 5: e4631.

https://doi.org/10.4236/oalib.1104631

Received: May 2, 2018

Accepted: June 23, 2018

Published: June 26, 2018

Copyright $\odot 2018$ by authors and Open Access Library Inc.

This work is licensed under the Creative Commons Attribution International License (CC BY 4.0).

http://creativecommons.org/licenses/by/4.0/

\begin{abstract}
On the whole real axis, we demonstrate sufficient conditions of regular solvability of third order operator-differential equations with complicated characteristics. These conditions were formulated only by the operator coefficients of the equation. In addition, by the principal part of the equation, the norms of the operators of intermediate derivative were estimated.
\end{abstract}

\section{Subject Areas \\ Ordinary Differential Equation}

\section{Keywords}

Operator-Differential Equation, Hilbert Space, Self-Adjoint Operator, Intermediate Derivative Operator

\section{Introduction}

In a separable Hilbert space $H$, we have the following equation:

$$
P u(x) \equiv p_{0} u(x)+p_{1} u(x)=f(x), x \in R,
$$

where

$$
\begin{aligned}
& p_{0} u(x)=\left(\frac{\mathrm{d}}{\mathrm{d} t}-A\right)\left(\frac{\mathrm{d}}{\mathrm{d} t}+A\right)^{2} u(x) . \\
& p_{1} u(x)=\sum_{s=1}^{2} A_{s} \frac{\mathrm{d}^{3-s} u(x)}{\mathrm{d} x^{3-s}},
\end{aligned}
$$

$A$ is a self-adjoint positive-definite operator, and $A_{s}, s=1,2$ are generally linear unbounded operators. All derivatives are understood in the sense of distribu- 
tions theory.

We consider $f(x) \in L_{2}(R ; H)$, where

$$
L_{2}(R ; H)=\left\{f(x):\|f(x)\|_{L_{2}(R ; H)}^{2}\left(\int_{-\infty}^{+\infty}\|f(x)\|_{H}^{2} \mathrm{~d} t\right)^{\frac{1}{2}}<+\infty\right\}
$$

(see [1] [2]), and $u(x) \in W_{2}^{3}(R ; H)$, which are determined as follows:

$$
W_{2}^{3}(R ; H)=\left\{u(x): \frac{\mathrm{d}^{3} u(x)}{\mathrm{d} x^{3}} \in L_{2}\left(R_{+} ; H\right), A^{3} u(x) \in L_{2}(R ; H)\right\} .
$$

With the norm

$$
\|u\|_{W_{2}^{3}\left(R_{+} ; H\right)}=\left(\left\|\frac{\mathrm{d}^{3} u}{\mathrm{~d} x^{3}}\right\|_{L_{2}(R ; H)}^{2}+\left\|A^{3} u\right\|_{L_{2}(R ; H)}^{2}\right)^{\frac{1}{2}},
$$

See [2].

Notice that the principal part of the investigated equation possesses complicated characteristic, not multiple characteristics as in [3].

Definition 1. If for any $f(x) \in L_{2}(R ; H)$ there exists a vector function $u(x) \in W_{2}^{2}(R ; H)$ that satisfies Equation (1) almost everywhere in $R$, then it is called a regular solution of Equation (1)

Definition 2. If for any $f(x) \in L_{2}(R ; H)$ there exists a regular solution of Equation (1), and satisfies the inequality

$$
\|u\|_{W_{2}^{3}(R ; H)} \leq \text { const }\|f\|_{L_{2}(R ; H)},
$$

then Equation (1) is called regularly solvable.

It is known that if $p_{1}: W_{2}^{3}(R ; H) \rightarrow L_{2}(R ; H)$, then $A^{3-s} \frac{\mathrm{d}^{s} u(x)}{\mathrm{d} x^{s}} \in L_{2}(R ; H)$, $s=1,2$.

And the following inequalities are valid (see [2]).

$$
\left\|A^{3-s} \frac{\mathrm{d}^{s} u(x)}{\mathrm{d} x^{s}}\right\|_{L_{2}(R ; H)} \leq c_{s}\|u\|_{W_{2}^{2}(R ; H)}, s=1,2 .
$$

Definition 3. Parseval's equality

$$
\int_{-\infty}^{+\infty}|f(x)|^{2} \mathrm{~d} x=\frac{1}{2 \pi} \int_{-\infty}^{+\infty}|\tilde{f}(\zeta)|^{2} \mathrm{~d} \zeta
$$

where,

$$
\tilde{f}(\zeta)=\int_{-\infty}^{+\infty} f(x) \mathrm{e}^{-i x \zeta} \mathrm{d} x
$$

\section{Main Results}

Theorem 1 . The operator $P_{0}$ is an isomorphism from the space $W_{2}^{3}(R ; H)$ to the space $L_{2}(R ; H)$.

Proof. From (2), it is easy to prove that the operator $P_{0}$ acts from $W_{2}^{3}(R ; H)$ to $L_{2}(R ; H)$ be bounded. Using Fourier transforms for the equation $P_{0} u(x)=f(x)$, we obtain 


$$
(-i \xi E-A)(-i \xi E+A)^{2} \tilde{u}(\xi)=\tilde{f}(\xi) .
$$

( $E$ is the unit operator), where $\tilde{u}(\xi), \tilde{f}(\xi)$ are Fourier transform for the functions $u(x), f(x)$, respectively. The operator pencil $(-i \xi E-A)(-i \xi E+A)^{2}$ is invertible and moreover

$$
\tilde{u}(\xi)=(-i \xi E-A)^{-1}(-i \xi E+A)^{-2} \tilde{f}(\xi),
$$

Hence,

$$
u(x)=\frac{1}{2 \pi} \int_{-\infty}^{+\infty}(-i \xi E-A)^{-1}(-i \xi E+A)^{-2} \tilde{f}(\xi) \mathrm{e}^{i \zeta x} \mathrm{~d} \zeta .
$$

We show that $u(x) \in W_{2}^{3}(R ; H)$. By using the Parseval equality and (3), we obtain:

$$
\begin{aligned}
\|u\|_{W_{2}^{3}(R ; H)}^{2}= & \left\|\frac{\mathrm{d}^{3} u}{\mathrm{~d} t^{3}}\right\|_{L_{2}(R ; H)}^{2}+\left\|A^{3} u\right\|_{L_{2}(R ; H)}^{2}=\left\|-i \zeta^{3} \tilde{u}(\xi)\right\|_{L_{2}(R ; H)}^{2}+\left\|A^{3} \tilde{u}(\xi)\right\|_{L_{2}(R ; H)}^{2} \\
= & \left\|-i \zeta^{3}(-i \xi E-A)^{-1}(-i \xi E+A)^{-2} \tilde{f}(\xi)\right\|_{L_{2}(R ; H)}^{2} \\
& +\left\|A^{3}(-i \xi E-A)^{-1}(-i \xi E+A)^{-2} \tilde{f}(\xi)\right\|_{L_{2}(R ; H)}^{2} \\
\leq & \sup _{\zeta \in R}\left\|-i \zeta^{3}(-i \zeta E-A)^{-1}(-i \zeta E+A)^{-2}\right\|_{H \rightarrow H}^{2}\|\tilde{f}(\zeta)\|_{L_{2}(R ; H)}^{2} \\
& +\sup _{\zeta \in R}\left\|A^{3}(-i \zeta E-A)^{-1}(-i \zeta E+A)^{-2}\right\|_{H \rightarrow H}^{2}\|\tilde{f}(\zeta)\|_{L_{2}(R ; H)}^{2}
\end{aligned}
$$

If $\sigma(A)$ is a spectrum of the operator $A$, then we consider

$$
\begin{aligned}
& \sup _{\xi \in R}\left\|-i \zeta^{3}(-i \zeta E-A)^{-1}(-i \zeta E+A)^{-2}\right\|_{H \rightarrow H} \\
& \leq \sup _{\xi \in R} \sup _{\sigma \in \sigma(A)}\left|-i \zeta^{3}(-i \zeta-\sigma)^{-1}(-i \zeta+\sigma)^{-2}\right| \\
& =\sup _{\xi \in R} \frac{|\zeta|^{3}}{\left(\zeta^{2}+\sigma^{2}\right)^{\frac{3}{2}}} \leq 1, \\
& \sup _{\xi \in R}\left\|A^{3}(-i \zeta E-A)^{-1}(i \zeta E+A)^{-2}\right\|_{H \rightarrow H} \\
& \leq \sup _{\xi \in R} \sup _{\sigma \in \sigma(A)}\left|\sigma^{3}(-i \zeta-\sigma)^{-1}(-i \zeta+\sigma)^{-2}\right| \\
& =\sup _{\sigma \in \sigma(A)} \frac{\sigma^{3}}{\left(\zeta^{2}+\sigma^{2}\right)^{\frac{3}{2}}} \leq 1
\end{aligned}
$$

Taking into account (5) and (6) into (4) we obtain:

$$
\|u\|_{W_{2}^{3}(R ; H)}^{2} \leq 2\|\bar{f}(\zeta)\|_{L_{2}(R ; H)}^{2}=2\|f(x)\|_{L_{2}(R ; H)}^{2} .
$$

Consequently, $u(x) \in W_{2}^{3}(R ; H)$.

Applying Banach theorem on the inverse operator, we get that the operator $P_{0}$ is an isomorphism from $W_{2}^{3}(R ; H)$ to $L_{2}(R ; H)$.

Now, we estimate the norms of intermediate derivative operators participating in the main part of the Equation (1) for finding exact conditions on regular sol- 
vability of the given equation, expressed only by its operator coefficients.

From theorem 1, we have that the norms $\left\|p_{0} u\right\|_{L_{2}(R ; H)}$ and $\|u\|_{W_{2}^{3}(R ; H)}$ are equivalent in the space $W_{2}^{3}(R ; H)$. Therefore by the norm $\left\|p_{0} u\right\|_{L_{2}(R ; H)}$ the theorem on intermediate derivatives is valid as well.

Theorem 2. Let $u(x) \in W_{2}^{3}(R ; H)$. Then there hold the following inequalities:

$$
\left\|A^{3-s} \frac{\mathrm{d}^{s} u(x)}{\mathrm{d} x^{s}}\right\|_{L_{2}(R ; H)} \leq a_{s}\left\|p_{0} u\right\|_{L_{2}(R ; H)}, s=1,2 .
$$

where $a_{1}=a_{2}=\frac{2}{3 \sqrt{3}}$.

Proof. To establish the validity of inequality (11) we make change $p_{0} u(x)=f(x)$ and apply the Fourier transformation. We get

$$
\begin{aligned}
& \left\|A^{3-s}(-i \zeta)^{s}(-i \zeta E-A)^{-1}(-i \zeta E+A)^{-2} \bar{f}(\zeta)\right\|_{L_{2}(R ; H)} \\
& \leq \sup _{\xi \in R}\left\|A^{3-s}(-i \zeta)^{s}(-i \zeta E-A)^{-1}(-i \zeta E+A)^{-2}\right\|_{H \rightarrow H}\|\bar{f}(\zeta)\|_{L_{2}(R ; H)}, s=1,2 .
\end{aligned}
$$

For $\zeta \in R$ we estimate the following norms:

$$
\begin{aligned}
& \left\|A^{3-s}(-i \zeta)^{s}(-i \zeta E-A)^{-1}(-i \zeta E+A)^{-2}\right\|_{H \rightarrow H} \\
& \leq \sup _{\sigma \in \sigma(A)}\left|\sigma^{3-s}(-i \zeta)^{s}(-i \zeta-\sigma)^{-1}(i \zeta+\sigma)^{-2}\right| \\
& =\sup _{\sigma \in \sigma(A)}\left|\sigma^{-s}(-i \zeta)^{s}\left(-i \frac{\zeta}{\sigma}-1\right)^{-1}\left(-i \frac{\zeta}{\sigma}+1\right)^{-2}\right| \\
& \leq \sup _{\mu=\frac{\xi^{2}}{\sigma^{2}}} \frac{\mu^{s / 2}}{(\mu+1)^{3 / 2}}=\frac{1}{3 \sqrt{3}} s^{s / 2}(3-s)^{(3-s) / 2}, s=1,2 .
\end{aligned}
$$

Finally, from (12), we have

$$
\left\|A^{3-s}(-i \zeta)^{s}(-i \zeta E-A)^{-1}(-i \zeta E+A)^{-2} \tilde{f}(\zeta)\right\|_{L_{2}(R ; H)} \leq a_{s}\|\tilde{f}(\xi)\|_{L_{2}(R ; H)}, s=1,2 .
$$

Lemma. The operator $P_{1}$ continuously acts from $W_{2}^{3}(R ; H)$ to $L_{2}(R ; H)$ provided that the operators $A_{s} A^{-s}, s=1,2$ are bounded in $H$.

Taking into account the results found up [4] to now we get possibility to establish regular solvability conditions of Equation (1).

Theorem 3. Let the operators $A_{s} A^{-s}, s=1,2$ be bounded in $\mathrm{H}$ and it holds the inequality $\sum_{s=1}^{2} a_{s}\left\|A_{3-s} A^{-(3-s)}\right\|_{H \rightarrow H} \prec 1$, where the numbers $a_{s}, s=1,2$ are determined in theorem 2. Then the Equation (1) is regularly solvable.

Proof. By theorem 1, provided that the operator $P_{0}$ has a bounded inverse operator $P_{0}^{-1}$ acting from $L_{2}(R ; H)$ to $W_{2}^{3}(R ; H)$, then after replacing $p_{0} u(x)=v(x)$ in Equation (1) can be written as $\left(E+p_{1} p_{0}^{-1}\right) v(x)=f(x)$.

Now we prove under the theorem conditions (see [5]), that the norm 


$$
\left\|p_{1} p_{0}^{-1}\right\|_{L_{2}(R ; H) \rightarrow L_{2}(R ; H)}<1 .
$$

By theorem (2), we have:

$$
\begin{aligned}
\left\|p_{1} p_{0}^{-1} v\right\|_{L_{2}(R ; H)} & =\left\|p_{1} u\right\|_{L_{2}(R ; H)} \leq \sum_{s=1}^{2}\left\|A^{s} \frac{\mathrm{d}^{3-s} u}{\mathrm{~d} x^{3-s}}\right\|_{L_{2}(R ; H)} \\
& \leq \sum_{s=1}^{2}\left\|A_{s} A^{-s}\right\|_{H \rightarrow H}\left\|A^{s} \frac{\mathrm{d}^{3-s} u}{\mathrm{~d} x^{3-s}}\right\|_{L_{2}(R ; H)} \\
& \leq \sum_{s=1}^{2} a_{3-s}\left\|A_{s} A^{-s}\right\|_{H \rightarrow H}\left\|p_{0} u\right\|_{L_{2}(R ; H)} \\
& =\sum_{s=1}^{2} a_{3-s}\left\|A_{s} A^{-s}\right\|_{H \rightarrow H}\|v\|_{L_{2}(R ; H)}
\end{aligned}
$$

Consequently,

$$
\left\|p_{1} p_{0}^{-1}\right\|_{L_{2}(R ; H) \rightarrow L_{2}(R ; H)} \leq \sum_{s=1}^{2} a_{3-s}\left\|A_{s} A^{-s}\right\|_{H \rightarrow H} \prec 1 .
$$

Thus, the operator $E+p_{1} p_{0}^{-1}$ is invertible in $L_{2}(R ; H)$ and hence $u(x)$ can be determined by $u(x)=p_{0}^{-1}\left(E+p_{1} p_{0}^{-1}\right)^{-1} f(x)$, moreover

$$
\begin{aligned}
\|u\|_{W_{2}^{3}(R ; H)} & \leq\left\|p_{0}^{-1}\right\|_{L_{2}(R ; H) \rightarrow W_{2}^{3}(R ; H)}\left\|\left(\left(E+p_{1} p_{0}^{-1}\right)\right)^{-1}\right\|_{L_{2}(R ; H) \rightarrow L_{2}(R ; H)}\|f\|_{L_{2}(R ; H)} \\
& \leq \text { const }\|f\|_{L_{2}(R ; H)} .
\end{aligned}
$$

The theorem is proved.

\section{Conclusion}

We formulated exact conditions on regular solvability of Equation (1), expressed only by its operator coefficients. We estimated the norms of intermediate derivative operators participating in the principle part of the given equation. In the case when in the perturbed part of Equation (1), the participant variable operator coefficients, i.e. $A_{s}(x), s=1,2$ are linear operators, which determined for all $x \in R$, are investigated in a similar way.

\section{References}

[1] Hille, E. and Phillips, R. (1962) Functional Analysis and Semi-Groups. IL, Moscow, 829 p. (In Russian)

[2] Lions, J.L. and Majenes, E. (1971) Inhomogeneous Boundary Value Problems and Their Applications. Mir, Moscow, 371 p. (In Russian)

[3] Aliev, A.R. and Elbably, A.L. (2012) On the Solvability in a Weight Space of a Third-Order Operator-Differential Equation with Multiple Characteristic. Doklady Mathematics, 85, 233-235. https://doi.org/10.1134/S106456241201036X

[4] Gasymov, M.G. and Mirzoev, S.S. (1992) On Solvability Boundary Value Problems for Elliptic Type Operator Differential Equations of Second Order. Different Uravnenie, 28, 651-666. (In Russian)

[5] Mirzoyev, S.S. (2003) On the Norms of Operators of Intermediate Derivatives, Transactions of NAS of Azerbaijan. Series Physical-Technical and Mathematical Sciences, 23, 157-164. 